\title{
ON PROJECTIONS OF SEMI-ALGEBRAIC SETS DEFINED BY FEW QUADRATIC INEQUALITIES
}

\author{
SAUGATA BASU AND THIERRY ZELL
}

\begin{abstract}
Let $S \subset \mathbb{R}^{k+m}$ be a compact semi-algebraic set defined by $P_{1} \geq$ $0, \ldots, P_{\ell} \geq 0$, where $P_{i} \in \mathbb{R}\left[X_{1}, \ldots, X_{k}, Y_{1}, \ldots, Y_{m}\right]$, and $\operatorname{deg}\left(P_{i}\right) \leq 2,1 \leq i \leq$ $\ell$. Let $\pi$ denote the standard projection from $\mathbb{R}^{k+m}$ onto $\mathbb{R}^{m}$. We prove that for any $q>0$, the sum of the first $q$ Betti numbers of $\pi(S)$ is bounded by $(k+m)^{O(q \ell)}$. We also present an algorithm for computing the the first $q$ Betti numbers of $\pi(S)$, whose complexity is $(k+m)^{2^{O(q \ell)}}$. For fixed $q$ and $\ell$, both the bounds are polynomial in $k+m$.
\end{abstract}

\section{INTRODUCTION}

Designing efficient algorithms for computing the Betti numbers of semi-algebraic sets is one of the outstanding open problems in algorithmic semi-algebraic geometry. There has been some recent progress in this area. It has been known for a while that the zero-th Betti number (which is also the number of connected components) of semi-algebraic sets can be computed in single exponential time. Very recently, it has been shown that even the first Betti number, and more generally the first $q$ Betti numbers for any fixed constant $q$, can be computed in single exponential time [10, 8]. Since the problem of deciding whether a given semi-algebraic set in $\mathbb{R}^{k}$ is empty or not is NP-hard, and that of computing its zero-th Betti number is \#P-hard, the existence of polynomial time algorithms for computing the Betti numbers is considered unlikely.

One particularly interesting case is that of semi-algebraic sets defined by quadratic inequalities. The class of semi-algebraic sets defined by quadratic inequalities is the first interesting class of semi-algebraic sets after sets defined by linear inequalities, in which case the problem of computing topological information reduces to linear programming for which (weakly) polynomial time algorithms are known. From the point of view of computational complexity, it is easy to see that the Boolean satisfiability problem can be posed as the problem of deciding whether a certain semi-algebraic set defined by quadratic inequalities is empty or not. Thus, deciding whether such a set is empty is clearly NP-hard and counting its number of connected components is \#P-hard. However, semi-algebraic sets defined by quadratic inequalities are distinguished from arbitrary semi-algebraic sets in the sense that, if the number of inequalities is fixed, then the sum of their Betti numbers is bounded polynomially in the dimension. The following bound was proved by Barvinok [3].

Key words and phrases. Betti numbers, Quadratic inequalities, Semi-algebraic sets, Spectral sequences, Cohomological descent.

The author was supported in part by an NSF Career Award 0133597 and a Sloan Foundation Fellowship. 
Theorem 1.1. Let $S \subset \mathbb{R}^{k}$ be a semi-algebraic set defined by the inequalities, $P_{1} \geq 0, \ldots, P_{\ell} \geq 0, \operatorname{deg}\left(P_{i}\right) \leq 2,1 \leq i \leq \ell$. Then, $\sum_{i=0}^{k} b_{i}(S) \leq k^{O(\ell)}$, where $b_{i}(S)$ denotes the $i$-th Betti number, which is the dimension of the $i$-th singular cohomology group of $S, H^{i}(S ; \mathbb{Q})$, with coefficients in $\mathbb{Q}$.

In view of Theorem 1.1 it is natural to consider the class of semi-algebraic sets defined by a fixed number of quadratic inequalities from a computational point of view. Algorithms for computing various topological properties of this class of semialgebraic sets have been developed, starting from the work of Barvinok [2], who described a polynomial time algorithm for testing emptiness of a set defined by a constant number of quadratic inequalities. This was later generalized and made constructive by Grigoriev and Pasechnik in [21], where an algorithm is described for computing sample points in every connected component of a semi-algebraic set defined over a quadratic map. More recently, polynomial time algorithms have been designed for computing the Euler-Poincaré characteristic [9] as well as all the Betti numbers [7] of sets defined by a fixed number of quadratic inequalities (with different dependence on the number of inequalities in the complexity bound). Note also that the problem of deciding the emptiness of a set defined by a single quartic equation is already NP-hard and hence it is unlikely that there exists polynomial time algorithms for any of the above problems if the degree is allowed to be greater than two.

A case of intermediate complexity between semi-algebraic sets defined by polynomials of higher degree and sets defined by a fixed number of quadratic sign conditions is obtained by considering projections of such sets. The operation of linear projection of semi-algebraic sets plays a very significant role in algorithmic semi-algebraic geometry. It is a consequence of the Tarski-Seidenberg principle (see for example 11, page 61) that the image of a semi-algebraic set under a linear projection is semi-algebraic, and designing efficient algorithms for computing properties of projections of semi-algebraic sets (such as its description by a quantifier-free formula) is a central problem of the area and is a very well-studied topic (see for example 31] or [11, Chapter 14). However, the complexities of the best algorithms for computing descriptions of projections of general semi-algebraic sets is singly exponential in the dimension and do not significantly improve when restricted to the class of semi-algebraic sets defined by a constant number of quadratic inequalities. Indeed, any semi-algebraic set can be realized as the projection of a set defined by quadratic inequalities, and it is not known whether quantifier elimination can be performed efficiently when the number of quadratic inequalities is kept constant. However, we show in this paper that, with a fixed number of inequalities, the projections of such sets are topologically simpler than projections of general semi-algebraic sets. This suggests, from the point of view of designing efficient (polynomial time) algorithms in semi-algebraic geometry, that projections of semi-algebraic sets defined by a constant number of quadratic inequalities is the next natural class of sets to consider, after sets defined by linear and (constant number of) quadratic inequalities, and this is what we proceed to do in this paper.

In this paper, we describe a polynomial time algorithm (Algorithm 2) for computing certain Betti numbers (including the zero-th Betti number which is the number of connected components) of projections of sets defined by a constant number of 
quadratic inequalities, without having to compute a semi-algebraic description of the projection. More precisely, let $S \subset \mathbb{R}^{k+m}$ be a compact semi-algebraic set defined by $P_{1} \geq 0, \ldots, P_{\ell} \geq 0$, with $P_{i} \in \mathbb{R}\left[X_{1}, \ldots, X_{k}, Y_{1}, \ldots, Y_{m}\right], \operatorname{deg}\left(P_{i}\right) \leq 2,1 \leq$ $i \leq \ell$. Let $\pi: \mathbb{R}^{k+m} \rightarrow \mathbb{R}^{m}$ be the projection onto the last $m$ coordinates. In what follows, the number of inequalities, $\ell$, used in the definition of $S$ will be considered as some fixed constant. Since, $\pi(S)$ is not necessarily describable using only quadratic inequalities, the bound in Theorem 1.1 does not hold for $\pi(S)$ and $\pi(S)$ can in principle be quite complicated. Using the best known complexity estimates for quantifier elimination algorithms over the reals (see [1]), we get single exponential (in $k$ and $m$ ) bounds on the degrees and the number of polynomials necessary to obtain a semi-algebraic description of $\pi(S)$. In fact, there is no known algorithm for computing a semi-algebraic description of $\pi(S)$ in time polynomial in $k$ and $m$. Nevertheless, we are able to prove that for any fixed constant $q>0$, the sum of the first $q$ Betti numbers of $\pi(S)$ are bounded by a polynomial in $k$ and $m$. More precisely, we obtain the following complexity bound (see Section 4).

Theorem 1.2. Let $S \subset \mathbb{R}^{k+m}$ be a compact semi-algebraic set defined by

$$
P_{1} \geq 0, \ldots, P_{\ell} \geq 0, P_{i} \in \mathbb{R}\left[X_{1}, \ldots, X_{k}, Y_{1}, \ldots, Y_{m}\right], \operatorname{deg}\left(P_{i}\right) \leq 2,1 \leq i \leq \ell .
$$

Let $\pi: \mathbb{R}^{k+m} \rightarrow \mathbb{R}^{m}$ be the projection onto the last $m$ coordinates. For any $q>$ $0,0 \leq q \leq k$

$$
\sum_{i=0}^{q} b_{i}(\pi(S)) \leq(k+m)^{O(q \ell)} .
$$

We also consider the problem of computing the Betti numbers of $\pi(S)$. Previously, there was no polynomial time algorithm for computing any non-trivial topological property of projections of sets defined by few quadratic inequalities. We describe a polynomial time algorithm for computing the first few Betti numbers of $\pi(S)$. The algorithm (Algorithm 2 in Section [7) computes $b_{0}(\pi(S)), \ldots, b_{q}(\pi(S))$. The complexity of the algorithm is $(k+m)^{2^{O(q \ell)}}$. If the coefficients of the input polynomials are integers of bit-size bounded by $\tau$, then the bit-size of the integers appearing in the intermediate computations and the output are bounded by $\tau(k+m)^{2^{O(q \ell)}}$. Note that the output of the algorithm includes $b_{0}(\pi(S))$, which is the number of connected components of $\pi(S)$. Alternatively, one could obtain $b_{0}(\pi(S)), \ldots, b_{q}(\pi(S))$ by computing a semi-algebraic description of $\pi(S)$ using an efficient quantifier elimination algorithm (such as the one described in [5]) and then using the algorithm described in [8] to compute the first few Betti numbers. However, the complexity of this method would be worse: single exponential in $k$ and $m$. Thus, our algorithm is able to compute efficiently non-trivial topological information about the projection, even though it does not compute a semi-algebraic description of that projection (it is not even known whether such a description could be computed in polynomial time).

In order to obtain Algorithm 2] we give a new construction of a certain spectral sequence, namely the cohomological descent spectral sequence, which plays a crucial role in the design of the algorithm. Even though variants of this spectral sequence have been known for some time [17, 32, 18, 24, 28, 34, to our knowledge this is the first time it has been used in designing efficient algorithms. The new construction that makes this possible is formally analogous to that of the Mayer-Vietoris spectral sequence, which has been used several times recently in designing algorithms for 
computing Betti numbers of semi-algebraic sets (see [6, 7, 8, 10]), and thus this new construction (see Proposition 5.3 below) might be of independent interest.

\section{Main IDEAS}

There are two main ingredients behind the results in this paper. The first is the use of cohomological descent, a spectral sequence first introduced by Deligne [17, 32 in the context of sheaf cohomology. This descent spectral sequence is used to compute the cohomology of the target of a continuous surjection (under certain hypotheses only, the limit of this spectral sequence is not, in general, the homology of the target). The first terms of the sequence are cohomology groups of certain fibered products over the surjection, and this allows to bound the Betti numbers of the target space in terms of the Betti numbers of those fibered products. This estimate was first used by Gabrielov, Vorobjov and Zell in [19] to give estimates on the Betti numbers of projections of semi-algebraic sets (and more generally, of semi-algebraic sets defined by arbitrary quantified formulas) without resorting to quantifier elimination. Another use of this sequence to establish upper-bounds can be found in 35] which contains effective estimates for the Betti numbers of semi-algebraic Hausdorff limits.

The most striking feature of this spectral sequence argument is that it enables one to deduce properties (for instance, bounds on the Betti numbers) of the projection of a set without having to explicitly describe the projection. For instance, consider a semi-algebraic subset of $R^{k}$ defined by a polynomial having a constant number (say $m$ ) of monomials (often referred to as a fewnomial). It is known due to classical results of Khovansky [25] (see also [4) that the Betti numbers of such sets can be bounded in terms of $m$ and $k$ independent of the degree of the polynomial. Using the spectral sequence argument mentioned above, it was proved in [19] that even the Betti numbers of the projection of such a set can be bounded in terms of the number of monomials, even though it is known (see 20]) that the projection itself might not admit a description in terms of fewnomials.

The construction of the descent spectral sequence given in [19] involves consideration of join spaces and their filtrations and is not directly amenable for algorithmic applications. In Section [5 we give an alternate construction of a descent spectral sequence. When applied to surjections between open sets this spectral sequence converges to the cohomology of the image. The proof of this fact is formally analogous to the proof of convergence of the spectral sequence arising from the generalized Mayer-Vietoris sequence. This new proof allows us to identify a certain double complex, whose individual terms corresponds to the chain groups of the fibered products of the original set. The fibered product (taken a constant number of times) of a set defined by few quadratic inequalities is again a set of the same type.

However, since there is no known algorithm for efficiently triangulating semialgebraic sets (even those defined by few quadratic inequalities) we cannot directly use the spectral sequence to actually compute the Betti numbers of the projections. In order to do that we need an additional ingredient. This second main ingredient is a polynomial time algorithm described in 7 for computing a complex whose cohomology groups are isomorphic to those of a given semi-algebraic set defined by a constant number of quadratic inequalities. Using this algorithm we are able 
to construct a certain double complex, whose associated total complex is quasiisomorphic to (implying having isomorphic homology groups) a suitable truncation of the one obtained from the cohomological descent spectral sequence mentioned above. This complex is of much smaller size and can be computed in polynomial time and is enough for computing the first $q$ Betti numbers of the projection in polynomial time for any fixed constant $q$.

The rest of the paper is organized as follows. In Section 3 we recall certain basic facts from algebraic topology including the notions of complexes, and double complexes of vector spaces and spectral sequences. We do not prove any results since all of them are quite classical and we refer the reader to appropriate references [15. 26. 11] for the proofs. In Section 4] we prove the estimate on the sum of Betti numbers (Theorem 1.2) of projections of semi-algebraic sets defined by quadratic inequalities. In Section [5 we give our new construction of the cohomological descent spectral sequence In Section [6] we briefly describe Algorithm 1 which is used to compute cohomology groups of semi-algebraic sets given by quadratic inequalities. This algorithm runs in polynomial time when the number of inequalities is constant. We only describe the inputs, outputs and the complexity estimates of the algorithms, referring the reader to 7 for more details. Finally, in Section $\mathbf{Z}$ we describe our algorithm (Algorithm 2) for computing the first few Betti numbers of projections of semi-algebraic sets defined by quadratic inequalities.

\section{Topological Preliminaries}

We first recall some basic facts from algebraic topology, related to double complexes, and spectral sequences associated to double complexes as well as to continuous maps between semi-algebraic sets. We refer the reader to [15, 26] for detailed proofs. We also fix our notations for these objects. All the facts that we need are well known, and we merely give a brief overview.

3.1. Complex of Vector Spaces. A cochain complex is a sequence $C^{\bullet}=\left\{C^{i} \mid i \in\right.$ $\mathbb{Z}\}$ of $\mathbb{Q}$-vector spaces together with a sequence of homomorphisms $\delta^{i}: C^{i} \rightarrow C^{i+1}$ for which $\delta^{i} \circ \delta^{i+1}=0$ for all $p$.

The cohomology groups, $H^{i}\left(C^{\bullet}\right)$ are defined by,

$$
H^{i}\left(C^{\bullet}\right)=Z^{i}\left(C^{\bullet}\right) / B^{i}\left(C^{\bullet}\right),
$$

where $B^{i}\left(C^{\bullet}\right)=\operatorname{Im}\left(\delta^{i-1}\right)$, and $Z^{i}\left(C^{\bullet}\right)=\operatorname{Ker}\left(\delta^{i}\right)$. The cohomology groups, $H^{*}\left(C^{\bullet}\right)$, are all $\mathbb{Q}$-vector spaces (finite dimensional if the vector spaces $C^{i}$ are themselves finite dimensional). We will henceforth omit reference to the field of coefficients $\mathbb{Q}$ which is fixed throughout the rest of the paper.

Given two complexes, $C^{\bullet}=\left(C^{i}, \delta^{i}\right)$ and $D^{\bullet}=\left(D^{i}, \partial^{i}\right)$, a homomorphism of complexes, $\phi: C^{\bullet} \rightarrow D^{\bullet}$, is a sequence of linear maps $\phi^{i}: C^{i} \rightarrow D^{i}$ verifying $\partial^{i} \circ \phi^{i}=\phi^{i+1} \circ \delta^{i}$ for all $i$.

In other words, the following diagram is commutative for all $i$.

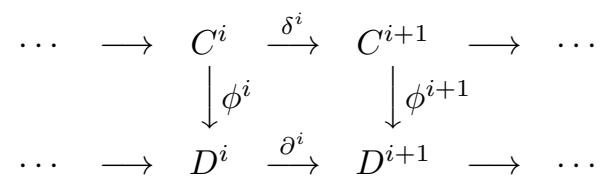


A homomorphism of complexes, $\phi: C^{\bullet} \rightarrow D^{\bullet}$, induces homomorphisms, $\phi^{*}$ : $H^{*}\left(C^{\bullet}\right) \rightarrow H^{*}\left(D^{\bullet}\right)$. The homomorphism $\phi$ is called a quasi-isomorphism if the homomorphisms $\phi^{*}$ are isomorphisms.

3.2. Double Complexes. A double complex is a bi-graded vector space

$$
C^{\bullet \bullet \bullet}=\bigoplus_{i, j \in \mathbb{Z}} C^{i, j},
$$

with co-boundary operators $d: C^{i, j} \rightarrow C^{i, j+1}$ and $\delta: C^{i, j} \rightarrow C^{i+1, j}$ such that $d^{2}=\delta^{2}=d \delta+\delta d=0$. We say that $C^{\bullet}, \bullet$ is a first quadrant double complex if it satisfies the condition that $C^{i, j}=0$ when $i j<0$.

Given a double complex $C^{\bullet \bullet \bullet}$, we can construct a complex $\operatorname{Tot}^{\bullet}\left(C^{\bullet}, \bullet\right)$, called the associated total complex of $C^{\bullet \bullet \bullet}$ and defined by $\operatorname{Tot}^{n}\left(C^{\bullet \bullet \bullet}\right)=\bigoplus_{i+j=n} C^{i, j}$, with differential $\mathcal{D}^{n}: \operatorname{Tot}^{n}\left(C^{\bullet \bullet \bullet}\right) \longrightarrow \operatorname{Tot}^{n+1}\left(C^{\bullet \bullet \bullet}\right)$ given by $D^{n}=d+\delta$.

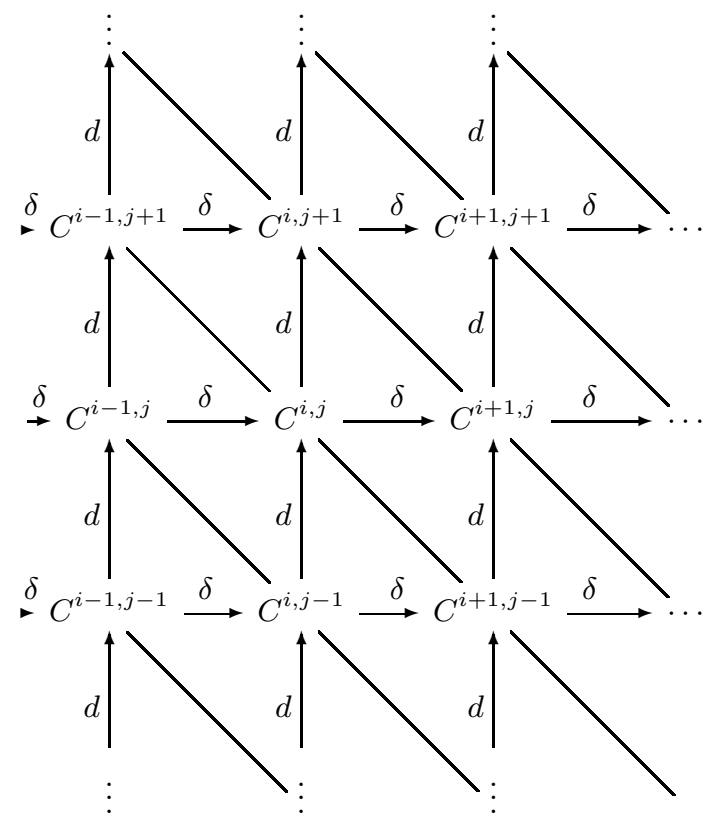

3.3. Spectral Sequences. A (cohomology) spectral sequence is a sequence of bigraded complexes $\left\{E_{r}^{i, j} \mid i, j, r \in \mathbb{Z}, r \geq a\right\}$ endowed with differentials $d_{r}^{i, j}: E_{r}^{i, j} \rightarrow$ $E_{r}^{i+r, j-r+1}$ such that $\left(d_{r}\right)^{2}=0$ for all $r$. Moreover, we require the existence of isomorphism between the complex $E_{r+1}$ and the homology of $E_{r}$ with respect to $d_{r}$ :

$$
E_{r+1}^{i, j} \cong H_{d_{r}}\left(E_{r}^{i, j}\right)=\frac{\operatorname{ker} d_{r}^{i, j}}{d_{r}^{i+r, j-r+1}\left(E_{r}^{i+r, j-r+1}\right)}
$$

The spectral sequence is called a first quadrant spectral sequence if the initial complex $E_{a}$ lies in the first quadrant, i.e. $E_{a}^{i, j}=0$ whenever $i j<0$. In that case, all subsequent complexes $E_{r}$ also lie in the first quadrant. Since the differential $d_{r}^{i, j}$ maps outside of the first quadrant for $r>i$, the homomorphisms of a first 


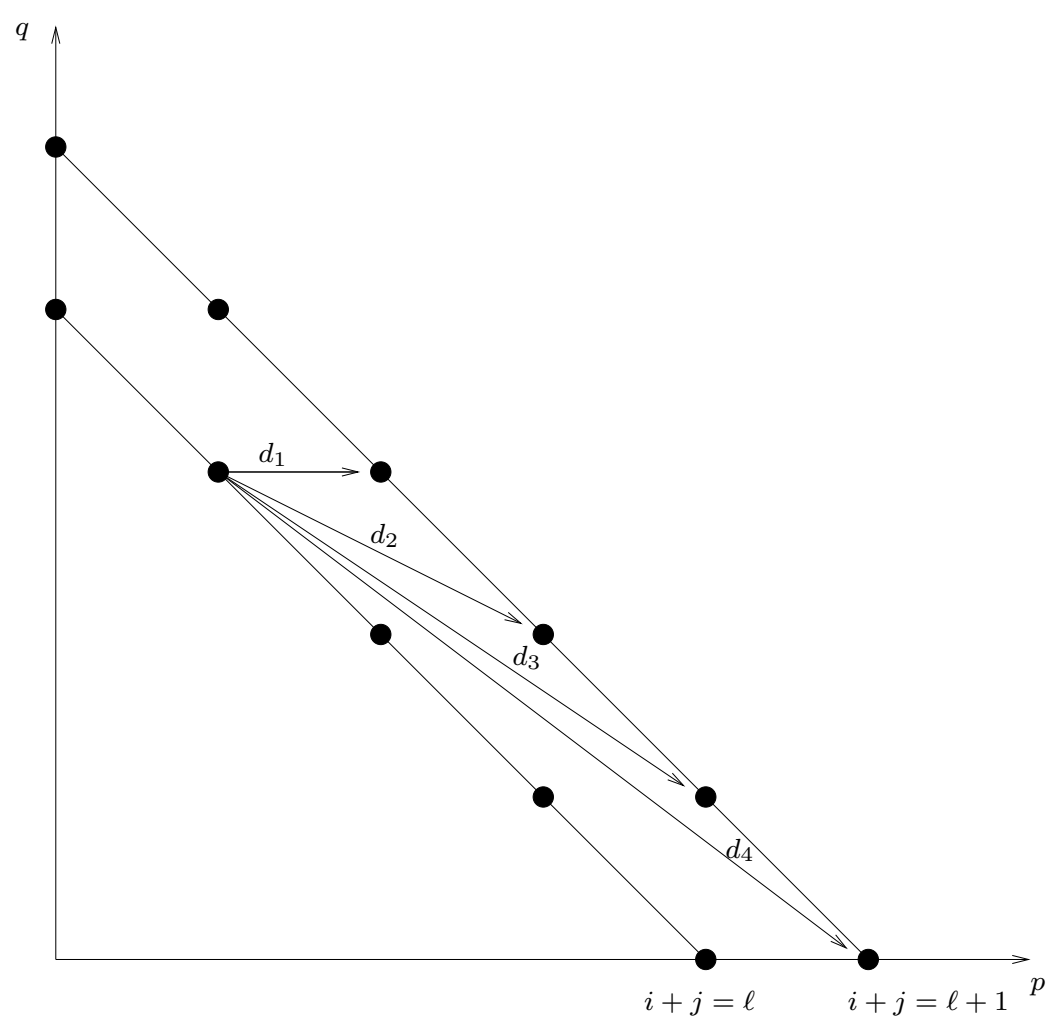

FiguRE 1. $d_{r}: E_{r}^{i, j} \rightarrow E_{r}^{i+r, j-r+1}$

quadrant spectral sequence $d_{r}$ are eventually zero, and thus the groups $E_{r}^{i, j}$ are all isomorphic to a fixed group $E_{\infty}^{i, j}$ for $r$ large enough, and we say the spectral sequence is convergent.

Given a double complex $C^{\bullet \bullet \bullet}$, we can associate to it two spectral sequences, ${ }^{\prime} E_{*}^{i, j},{ }^{\prime \prime} E_{*}^{i, j}$ (corresponding to taking row-wise or column-wise filtrations respectively).

If the double complex lies in the first quadrant, both of these spectral sequences are first quadrant spectral sequence, and both converge to $H^{*}\left(\operatorname{Tot}^{\bullet}\left(C^{\bullet \bullet \bullet}\right)\right)$, meaning that the limit groups verify

$$
\bigoplus_{i+j=n}{ }^{\prime} E_{\infty}^{i, j} \cong \bigoplus_{i+j=n}{ }^{\prime \prime} E_{\infty}^{i, j} \cong H^{n}\left(\operatorname{Tot}^{\bullet}\left(C^{\bullet, \bullet}\right)\right)
$$

for each $n \geq 0$.

The first terms of these are ${ }^{\prime} E_{1}=H_{\delta}\left(C^{\bullet \bullet \bullet}\right),{ }^{\prime} E_{2}=H_{d} H_{\delta}\left(C^{\bullet \bullet \bullet}\right)$, and ${ }^{\prime \prime} E_{1}=$ $H_{d}\left(C^{\bullet \bullet \bullet}\right),{ }^{\prime \prime} E_{2}=H_{\delta} H_{d}\left(C^{\bullet \bullet \bullet}\right)$.

Given two (first quadrant) double complexes, $C^{\bullet \bullet \bullet}$ and $\bar{C}^{\bullet \bullet \bullet}$, a homomorphism of double complexes $\phi: C^{\bullet} \bullet \rightarrow \bar{C}^{\bullet}, \bullet$ is a collection of homomorphisms, $\phi^{i, j}: C^{i, j} \rightarrow$ $\bar{C}^{i, j}$, such that the following diagrams commute. 


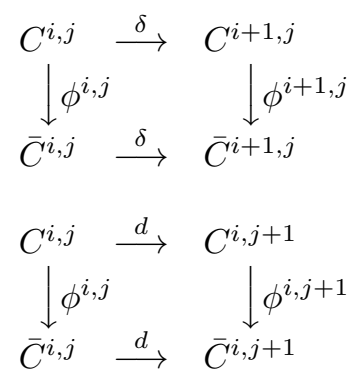

A homomorphism of double complexes, $\phi: C^{\bullet, \bullet} \rightarrow \bar{C}^{\bullet \bullet \bullet}$ induces homomorphisms $\phi_{r}^{i, j}: E_{r}^{i, j} \rightarrow \bar{E}_{r}^{i, j}$ between the terms of the associated spectral sequences (corresponding either to the row-wise or column-wise filtrations).

We will need the following useful fact (see [26], page 66, Theorem 3.4 for a proof).

Theorem 3.1. If $\phi_{s}^{i, j}$ is an isomorphism for some $s \geq 1$ (and all $i, j$ ), then $E_{r}^{i, j}$ and $\bar{E}_{r}^{i, j}$ are isomorphic for all $r \geq s$. In other words, the induced homomorphism, $\phi: \operatorname{Tot}^{\bullet}\left(C^{\bullet} \bullet\right) \longrightarrow \operatorname{Tot}^{\bullet}\left(\bar{C}^{\bullet}, \bullet\right)$ is a quasi-isomorphism.

\section{Proof of Theorem 1.2}

The proof of Theorem 1.2 relies on the bounds from Theorem 1.1 and on the following theorem that appears in [19.

Theorem 4.1. Let $X$ and $Y$ be two semi-algebraic sets and $f: X \rightarrow Y$ a semialgebraic continuous surjection such that $f$ is closed. Then for any integer $n$, we have

$$
b_{n}(Y) \leq \sum_{i+j=n} b_{j}\left(W_{f}^{i}(X)\right)
$$

where $W_{f}^{i}(X)$ denotes the $(i+1)$-fold fibered product of $X$ over $f$ :

$$
W_{f}^{i}(X)=\left\{\left(\bar{x}_{0}, \ldots, \bar{x}_{i}\right) \in X^{i+1} \mid f\left(\bar{x}_{0}\right)=\cdots=f\left(\bar{x}_{i}\right)\right\} .
$$

This theorem follows from the existence of a spectral sequence $E_{r}^{i, j}$ converging to $H^{*}(Y)$ and such that $E_{1}^{i, j} \cong H^{j}\left(W_{f}^{i}(X)\right)$. Since, in any spectral sequence, the dimensions of the terms $E_{r}^{i, j}$ are decreasing when $i$ and $j$ are fixed and $r$ increases, we obtain using the definition (3.1) of convergence:

$$
b_{n}(Y)=\sum_{i+j=n} \operatorname{dim}\left(E_{\infty}^{i, j}\right) \leq \sum_{i+j=n} \operatorname{dim}\left(E_{1}^{i, j}\right),
$$

yielding inequality (4.1).

The spectral sequence $E_{r}^{i, j}$, known as cohomological descent, originated with the work of Deligne [17, 32, in the framework of sheaf cohomology. In [19, the sequence is obtained as the spectral sequence associated to the filtration of an infinite dimensional topological object, the join space, constructed from $f$. For the purposes of Algorithm 2 we will give a different construction of this sequence (see Section (5). 
Proof of Theorem 1.2. Since $S$ is compact, the semi-algebraic continuous surjection $\pi: S \rightarrow \pi(S)$ is closed: applying Theorem 4.1 to $\pi$, inequality (4.1) yields for each $n$ with $0 \leq n \leq q$,

$$
b_{n}(\pi(S)) \leq \sum_{i+j=n} b_{j}\left(W_{\pi}^{i}(S)\right) .
$$

Notice that $W_{\pi}^{i}(S)=\left\{\left(\bar{x}_{0}, \ldots, \bar{x}_{i}, y\right) \mid P_{h}\left(\bar{x}_{t}, y\right) \geq 0,1 \leq h \leq \ell, 0 \leq t \leq i\right\}$. Thus, each $W_{\pi}^{i}(S) \subset \mathbb{R}^{(i+1) k+m}$ is defined by $\ell(i+1)$ quadratic inequalities. Applying the bound in Theorem 1.1 we get that,

$$
b_{j}\left(W_{\pi}^{i}(S)\right) \leq((i+1) k+m)^{O(\ell(i+1))} .
$$

Using inequalities (4.2) and (4.3) we get that,

$$
\sum_{i=0}^{q} b_{i}(\pi(S)) \leq(k+m)^{O(q \ell)},
$$

which proves the theorem.

\section{Cohomological Descent}

This section is devoted a new construction of the cohomological descent spectral sequence (already discussed in Section 4). In Theorem [5.6] we obtain this sequence as the spectral sequence associated to a double complex associated to the fibered powers of $X$, rather than through the filtration of the join space. Convergence to the cohomology of the target space occurs when the map $f: X \rightarrow Y$ is locally split (see definition below). By deformation, we are able to extend the result to our case of interest: the projection of a compact semi-algebraic set (Corollary [5.8).

We will use this construction for Algorithm 2

Definition 5.1. A continuous surjection $f: X \rightarrow Y$ is called locally split if there exists an open covering $\mathcal{U}$ of $Y$ such that for all $U \in \mathcal{U}$, there exists a continuous section $\sigma: U \rightarrow X$ of $f$, i.e. $\sigma$ is a continuous map such that $f(\sigma(y))=y$ for all $y \in U$.

In particular, if $X$ is an open semi-algebraic set and $f: X \rightarrow Y$ is a projection, the map $f$ is obviously locally split. This specific case is what we will use in Algorithm [2] as we will reduce the projection of compact semi-algebraic sets to projections of open semi-algebraic sets (see Proposition 5.7) in order to apply the spectral sequence.

Recall that for any semi-algebraic surjection $f: X \rightarrow Y$, we denoted by $W_{f}^{p}(X)$ the $(p+1)$-fold fibered power of $X$ over $f$,

$$
W_{f}^{p}(X)=\left\{\left(\bar{x}_{0}, \ldots, \bar{x}_{p}\right) \in X^{p+1} \mid f\left(\bar{x}_{0}\right)=\cdots=f\left(\bar{x}_{p}\right)\right\} .
$$

The map $f$ induces for each $p \geq 0$, a map from $W_{f}^{p}(X)$ to $Y$, sending $\left(\bar{x}_{0}, \ldots, \bar{x}_{p}\right)$ to the common value $f\left(\bar{x}_{0}\right)=\cdots=f\left(\bar{x}_{p}\right)$, and abusing notations a little we will denote this map by $f$ as well. 
5.1. Singular (co-)homology. We recall here the basic definitions related to singular (co-)homology theory directing the reader to 23] for details.

For any semi-algebraic set $X$, let $C \cdot(X)$ denote the complex of singular chains of $X$ with boundary map denoted by $\partial$.

Recall that $C_{\bullet}(X)$ is defined as follows: For $m \geq 0$, a singular $m$-simplex $s$ is a continuous map, $s: \Delta_{m} \rightarrow X$, where $\Delta_{m}$ is the standard $m$-dimensional simplex defined by,

$$
\Delta_{m}=\left\{\left(t_{0}, \ldots, t_{m}\right) \mid t_{i} \geq 0, \sum_{i=0}^{m} t_{i}=1\right\} .
$$

$C_{m}(X)$ is the vector space spanned by all singular $m$-simplices with boundary maps defined as follows. As usual we first define the face maps

$$
f_{m, i}: \Delta_{m} \rightarrow \Delta_{m+1}
$$

by $f_{m, i}\left(\left(t_{0}, \ldots, t_{m}\right)\right)=\left(t_{0}, \ldots, t_{i-1}, 0, t_{i+1}, \ldots, t_{m+1}\right)$.

For a singular $m$-simplex $s$ we define

$$
\partial s=\sum_{i=0}^{m}(-1)^{i} s \circ f_{m-1, i} .
$$

and extend $\partial$ to $C_{m}(X)$ by linearity. We will denote by $C^{\bullet}(X)$ the dual complex and by $d$ the corresponding co-boundary map. More precisely, given $\phi \in C^{m}(X)$, and a singular $(m+1)$-simplex $s$ of $X$, we have

$$
d \phi(s)=\sum_{i=0}^{m+1}(-1)^{i} \phi\left(s \circ f_{m, i}\right) .
$$

If $f: X \rightarrow Y$ is a continuous map, then it naturally induces a homomorphism $f_{*}: C_{\bullet}(X) \rightarrow C_{\bullet}(Y)$ by defining, for each singular $m$-simplex $s: \Delta_{m} \rightarrow X$, $f_{*}(s)=s \circ f: \Delta_{m} \rightarrow Y$, which is a singular $m$-simplex of $Y$. We will denote by $f^{*}: C^{\bullet}(Y) \rightarrow C^{\bullet}(X)$ the dual homomorphism. More generally, suppose that $s=\left(s_{0}, \ldots, s_{p}\right): \Delta_{m} \rightarrow W_{f}^{p}(X)$ is a singular $m$-simplex of $W_{f}^{p}(X)$. Notice that each component, $s_{i}, 0 \leq i \leq p$ are themselves singular $m$-simplices of $X$ and that $f_{*}\left(s_{0}\right)=\ldots=f_{*}\left(s_{p}\right)$ are equal as singular $m$-simplices of $Y$. We will denote their common image by $f_{*}(s)$.

We will require the notion of small simplices subordinate to an open covering of a topological space (see [23]). Assuming that $f: X \rightarrow Y$ is locally split, let $\mathcal{U}$ be an open covering of $Y$ on which local continuous sections exist. We denote by $\mathcal{V}$ the open covering of $X$ given by the inverse images of elements of $\mathcal{U}$, i.e. $\mathcal{V}=$ $\left\{f^{-1}(U) \mid U \in \mathcal{U}\right\}$. We let $C_{\bullet}^{\mathcal{U}}(Y)$ be the subcomplex of $C_{\bullet}(Y)$ spanned by those singular simplices of $Y$ whose images are contained in some element of the cover $\mathcal{U}$. Similarly, we let $C_{\bullet} \mathcal{V}(X)$ be the subcomplex of $C_{\bullet}(X)$ spanned by the simplices of $X$ with image in $\mathcal{V}$, and more generally, for any integer $p, C_{\bullet}^{\mathcal{V}}\left(W_{f}^{p}(X)\right)$ denotes the subcomplex of $C_{\bullet}\left(W_{f}^{p}(X)\right)$ spanned by simplices with image contained in $V^{p+1}$ for some $V \in \mathcal{V}$. The corresponding dual cochain complexes will be denoted by $C_{\mathcal{U}}^{\bullet}(Y)$ and $C_{\mathcal{V}}\left(W_{f}^{p}(X)\right)$ respectively. We will henceforth call any singular simplex of $C_{\bullet}^{\mathcal{U}}(Y)$ and any singular simplex of $C_{\bullet}^{\mathcal{V}}\left(W_{f}^{p}(X)\right)$ admissible simplices.

The inclusion homomorphism, $\iota_{\bullet}: C_{\bullet}^{\mathcal{U}}(Y) \hookrightarrow C_{\bullet}(Y)$ induces a dual homomorphism, $\iota^{\bullet}: C^{\bullet}(Y) \rightarrow C_{\mathcal{U}}^{\bullet}(Y)$. We also have corresponding induced homomorhisms, $\iota^{\bullet}: C^{\bullet}\left(W_{f}^{p}(X)\right) \rightarrow C_{\mathcal{V}}^{\bullet}\left(W_{f}^{p}(X)\right)$ for each $p \geq 0$. 
Proposition 5.2. The homomorphism $\iota^{\bullet}: C^{\bullet}(Y) \rightarrow C_{\mathcal{U}}^{\bullet}(Y)\left(\operatorname{resp} . C^{\bullet}\left(W_{f}^{p}(X)\right) \rightarrow\right.$ $C_{\mathcal{V}}^{\bullet}\left(W_{f}^{p}(X)\right)$ for each $\left.p \geq 0\right)$ is a chain homotopy equivalence. In particular, we have $H^{*}\left(C_{\mathcal{U}}^{\bullet}(Y)\right) \cong H^{*}\left(C^{\bullet}(Y)\right) \cong H^{*}(Y)$ and $H^{*}\left(C_{\mathcal{V}}^{\bullet}\left(W_{f}^{p}(X)\right)\right) \cong H^{*}\left(C^{\bullet}\left(W_{f}^{p}(X)\right)\right) \cong$ $H^{*}\left(W_{f}^{p}(X)\right)$.

Proof. This follows from a similar result for homology, see Proposition 2.21 in [23].

5.2. A long exact sequence. For each $p \geq 0$, we now define a homomorphism,

$$
\delta^{p}: C^{\bullet}\left(W_{f}^{p}(X)\right) \longrightarrow C^{\bullet}\left(W_{f}^{p+1}(X)\right)
$$

as follows: for each $i, 0 \leq i \leq p$, define $\pi_{p, i}: W_{f}^{p}(X) \rightarrow W_{f}^{p-1}(X)$ by,

$$
\pi_{p, i}\left(x_{0}, \ldots, x_{p}\right)=\left(x_{0}, \ldots, \widehat{x_{i}}, \ldots, x_{p}\right)
$$

( $\pi_{p, i}$ drops the $i$-th coordinate).

We will denote by $\left(\pi_{p, i}\right)_{*}$ the induced map on $C_{\bullet}\left(W_{f}^{p}(X)\right) \rightarrow C_{\bullet}\left(W_{f}^{p-1}(X)\right)$ and let $\pi_{p, i}^{*}: C^{\bullet}\left(W_{f}^{p-1}(X)\right) \rightarrow C^{\bullet}\left(W_{f}^{p}(X)\right)$ denote the dual map. For $\phi \in C^{\bullet}\left(W_{f}^{p}(X)\right)$, we define $\delta^{p} \phi$ by,

$$
\delta^{p} \phi=\sum_{i=0}^{p+1}(-1)^{i} \pi_{p+1, i}^{*} \phi .
$$

Note that for any open covering $\mathcal{V}$ of $X$, the map $\delta^{p}$ induces by restriction a map $C_{\mathcal{V}}\left(W_{f}^{p}(X)\right) \rightarrow C_{\mathcal{V}}\left(W_{f}^{p+1}(X)\right)$ which we will still denote by $\delta^{p}$.

The following proposition is analogous to the exactness of the generalized MayerVietoris sequence (cf. Lemma 1 in [6]).

Proposition 5.3. Let $f: X \rightarrow Y$ be a continuous, locally split surjection, where $X$ and $Y$ are semi-algebraic subsets of $\mathbb{R}^{n}$ and $\mathbb{R}^{m}$ respectively. Let $\mathcal{U}$ denote an open covering of $Y$ in which continuous sections of $f$ can be defined on every $U \in \mathcal{U}$, and $\mathcal{V}$ denote the open covering of $X$ obtained by inverse image of $\mathcal{U}$ under $f$. The following sequence is exact.

$$
\begin{gathered}
0 \longrightarrow C_{\mathcal{U}}^{\bullet}(Y) \stackrel{f^{*}}{\longrightarrow} C_{\mathcal{V}}^{\bullet}\left(W_{f}^{0}(X)\right) \stackrel{\delta^{0}}{\longrightarrow} C_{\mathcal{V}}^{\bullet}\left(W_{f}^{1}(X)\right) \stackrel{\delta^{1}}{\longrightarrow} \cdots \\
\quad \ldots \stackrel{\delta^{p-1}}{\longrightarrow} C_{\mathcal{V}}^{\bullet}\left(W_{f}^{p}(X)\right) \stackrel{\delta^{p}}{\longrightarrow} C_{\mathcal{V}}^{\bullet}\left(W_{f}^{p+1}(X)\right) \stackrel{\delta^{p+1}}{\longrightarrow} \cdots
\end{gathered}
$$

Proof. We will start by treating separately the first two positions in the sequence, then prove exactness for $p \geq 1$.

(A) $f^{*}: C_{\mathcal{U}}^{\bullet}(Y) \rightarrow C_{\mathcal{V}}^{\bullet}(X)$ is injective.

Let $U \in \mathcal{U}$ and let $s$ be a simplex whose image is contained in $U$. If $\sigma$ is a continuous section of $f$ defined on $U$, the simplex $t=\sigma_{*}(s)$ is in $C_{\bullet}^{\mathcal{V}}(X)$, and verifies $f_{*}(t)=s$. Hence, $f_{*}: C_{\bullet}^{\mathcal{V}}(X) \rightarrow C_{\bullet}^{\mathcal{U}}(Y)$ is surjective, so $f^{*}$ is injective.

(B) $f^{*}\left(C_{\mathcal{U}}^{\bullet}(Y)\right)=\operatorname{ker} \delta^{0}$.

Let $\phi \in C_{\mathcal{V}}^{m}(X)$. Any simplex $s \in C_{m}^{\mathcal{V}}\left(W_{f}^{1}(X)\right)$ is a pair $\left(s_{0}, s_{1}\right)$ of simplices in $C_{m}^{\mathcal{V}}(X)$ verifying $f_{*}\left(s_{0}\right)=f_{*}\left(s_{1}\right)$. We then have $\delta^{0} \phi(s)=$ $\phi\left(s_{1}\right)-\phi\left(s_{0}\right)$. If $\phi=f^{*} \psi$ for some $\psi \in C_{\mathcal{U}}^{m}(Y)$, we have for any $s$,

$$
\delta^{0} \phi(s)=f^{*} \psi\left(s_{1}\right)-f^{*} \psi\left(s_{0}\right)=\psi\left(f_{*}\left(s_{1}\right)\right)-\psi\left(f_{*}\left(s_{0}\right)\right)=0,
$$


since we must have $f_{*}\left(s_{0}\right)=f_{*}\left(s_{1}\right)$. Thus, we have $f^{*}\left(C_{\mathcal{U}}(Y)\right) \subset \operatorname{ker} \delta^{0}$.

Conversely, if $\phi$ is such that $\delta^{0} \phi=0$, this means that for any pair $\left(s_{0}, s_{1}\right)$ of simplices in $C_{m}^{\mathcal{V}}(X)$ verifying $f_{*}\left(s_{0}\right)=f_{*}\left(s_{1}\right)$, we have $\phi\left(s_{0}\right)=\phi\left(s_{1}\right)$. Since we just proved in part (A) that $f_{*}: C_{\bullet}^{\mathcal{V}}(X) \rightarrow C_{\bullet}^{\mathcal{U}}(Y)$ is surjective, any element $t \in C_{m}^{\mathcal{U}}(Y)$ is of the form $t=f_{*}(s)$ for some $s \in C_{m}^{\mathcal{V}}(X)$. Thus, we can define $\psi \in C_{\mathcal{U}}^{m}(Y)$ by $\psi(t)=\phi(s)$, and the condition $\delta^{0} \phi=0$ ensures that $\psi$ is well defined since its value does not depend on the choice of $s$ in the representation $t=f_{*}(s)$. This yields the reverse inclusion, and hence exactness at $p=0$.

(C) $\delta^{p+1} \circ \delta^{p}=0$ :

From the definitions of the maps $\pi_{p+1, i}^{*}, \pi_{p+2, j}^{*}$ we have that for $0 \leq i \leq$ $p+1,0 \leq j \leq p+2$,

$$
\pi_{p+2, j}^{*} \circ \pi_{p+1, i}^{*}(\phi)=\pi_{p+2, i+1}^{*} \circ \pi_{p+1, j}^{*}(\phi) \text { if } j<i .
$$

Let $\phi \in C_{\mathcal{V}}^{m}\left(W_{f}^{p}(X)\right)$. Now from the definitions of $\delta^{p}$ and $\delta^{p+1}$ we have that,

$$
\begin{aligned}
\delta^{p+1} \circ \delta^{p}(\phi) & =\delta^{p+1}\left(\sum_{i=0}^{p+1}(-1)^{i} \pi_{p+1, i}^{*}(\phi)\right) ; \\
& =\sum_{i=0}^{p+1}(-1)^{i} \delta^{p+1}\left(\pi_{p+1, i}^{*}(\phi)\right) ; \\
& =\sum_{i=0}^{p+1} \sum_{j=0}^{p+2}(-1)^{i+j} \pi_{p+2, j}^{*} \circ \pi_{p+1, i}^{*}(\phi) ; \\
& =\sum_{i=0}^{p+1}\left[\sum_{0 \leq j<i}(-1)^{i+j} \pi_{p+2, j}^{*} \circ \pi_{p+1, i}^{*}(\phi)+\sum_{i \leq j \leq p+2}(-1)^{i+j} \pi_{p+2, j}^{*} \circ \pi_{p+1, i}^{*}(\phi)\right] \\
& \left.=\sum_{i \leq j}(-1)^{i+j} \pi_{p+2, j}^{*} \circ \pi_{p+1, i}^{*}(\phi)+\sum_{i>j}(-1)^{i+j} \pi_{p+2, j}^{*} \circ \pi_{p+1, i}^{*}(\phi)\right) .
\end{aligned}
$$

Now using Equation (5.4), the previous line becomes

$$
\left.=\sum_{i \leq j}(-1)^{i+j} \pi_{p+2, j}^{*} \circ \pi_{p+1, i}^{*}(\phi)+\sum_{i>j}(-1)^{i+j} \pi_{p+2, i+1}^{*} \circ \pi_{p+1, j}^{*}(\phi)\right) .
$$

Interchanging $i$ and $j$ in the second summand of the previous line, we get

$$
\left.=\sum_{i \leq j}(-1)^{i+j} \pi_{p+2, j}^{*} \circ \pi_{p+1, i}^{*}(\phi)+\sum_{i<j}(-1)^{i+j} \pi_{p+2, j+1}^{*} \circ \pi_{p+1, i}^{*}(\phi)\right) .
$$

Finally, replacing $j+1$ by $j$ in the second summand above, we obtain

$$
\left.=\sum_{i \leq j}(-1)^{i+j} \pi_{p+2, j}^{*} \circ \pi_{p+1, i}^{*}(\phi)+\sum_{i<j-1}(-1)^{i+j-1} \pi_{p+2, j}^{*} \circ \pi_{p+1, i}^{*}(\phi)\right) ;
$$


and isolating the terms corresponding to $j=i$ and $j=i+1$ in the first sum gives

$$
\begin{aligned}
= & (-1)^{2 i} \pi_{p+2, i}^{*} \circ \pi_{p+1, i}^{*}(\phi)+(-1)^{2 i+1} \pi_{p+2, i}^{*} \circ \pi_{p+1, i+1}^{*}(\phi) \\
& \left.+\sum_{i<j-1}(-1)^{i+j} \pi_{p+2, j}^{*} \circ \pi_{p+1, i}^{*}(\phi)+\sum_{i<j-1}(-1)^{i+j-1} \pi_{p+2, j}^{*} \circ \pi_{p+1, i}^{*}(\phi)\right) ; \\
= & 0 ;
\end{aligned}
$$

(since, again, by Equation (5.4), we have $\pi_{p+2, i}^{*} \circ \pi_{p+1, i+1}^{*}=\pi_{p+2, i}^{*} \circ \pi_{p+1, i}^{*}$ ).

(D) $\operatorname{Im}\left(\delta^{p}\right) \supset \operatorname{Ker}\left(\delta^{p+1}\right)$ :

Let $\phi \in \operatorname{Ker}\left(\delta^{p+1}\right)$. In other words, for each admissible singular $m$-simplex $s=\left(s_{0}, \ldots, s_{p+1}\right): \Delta_{m} \rightarrow W_{f}^{p+2}(X)$

$$
\sum_{i=0}^{p+2}(-1)^{i} \phi\left(\left(s_{0}, \ldots, \hat{s}_{i}, \ldots, s_{p+2}\right)\right)=0 .
$$

For each admissible singular $m$-simplex $s$ of $Y$ let $s_{*}$ denote denote a fixed admissible singular $m$-simplex of $X$ such that $f_{*}\left(s_{*}\right)=s$. Such a choice is possible since, as we proved in part $(\mathrm{A}), f_{*}$ is surjective onto $C_{\bullet}^{\mathcal{U}}(Y)$. Let $\psi \in C_{\mathcal{V}}^{m}\left(W_{f}^{p}(X)\right)$ be defined as follows. For an admissible singular $m$-simplex $t=\left(t_{0}, \ldots, t_{p}\right)$ of $W_{f}^{p}(X)$ we define

$$
\psi(t)=\phi\left(f_{*}(t), t_{0}, \ldots, t_{p}\right) .
$$

Now for an admissible singular $m$ simplex $t=\left(t_{0}, \ldots, t_{p+1}\right)$ of $W_{f}^{p+1}$, with

$$
\begin{aligned}
\delta^{p} \psi(t) & =\sum_{i=0}^{p+1}(-1)^{i} \pi_{p+1, i}^{*} \psi(t) \\
& =\sum_{i=0}^{p+1}(-1)^{i} \psi\left(\left(t_{0}, \ldots, \hat{t}_{i}, \ldots, t_{p+1}\right)\right) \\
& =\sum_{i=0}^{p+1}(-1)^{i} \phi\left(\left(f_{*}(t), t_{0}, \ldots, \hat{t}_{i}, \ldots, t_{p+1}\right)\right)
\end{aligned}
$$

Now let $s$ denote the admissible singular $m$-simplex of $W_{f}^{p+2}(X)$ defined by $s=\left(f_{*}(t), t_{0}, \ldots, \hat{t}_{i}, \ldots, t_{p+1}\right)$. Now applying Equation (5.5), we get

$$
\sum_{i=0}^{p+2}(-1)^{i} \phi\left(\left(s_{0}, \ldots, \widehat{s_{i}}, \ldots, s_{p+2}\right)\right)=0 .
$$

Separating the first term from the rest we obtain,

$$
\phi\left(\left(t_{0}, \ldots, t_{p+1}\right)=\sum_{i=0}^{p+1}(-1)^{i} \phi\left(\left(f_{*}(t), t_{0}, \ldots, \hat{t}_{i}, \ldots, t_{p+1}\right)\right)=\delta^{p} \psi(t) .\right.
$$

This finally proves the exactness of the sequence. 
5.3. The descent double complex. Now, let $D^{\bullet \bullet} \bullet(X)$ denote the double complex defined by, $D^{p, q}(X)=C^{q}\left(W_{f}^{p}(X)\right)$ with vertical and horizontal homomorphisms given by $\tilde{d}^{q}=(-1)^{p} d^{q}$ and $\delta$ respectively, where $d$ is the singular coboundary operator (5.2) and $\delta$ is the map defined in (5.3). Also, let $D^{p, q}(X)=0$ if $p<0$ or $q<0$.

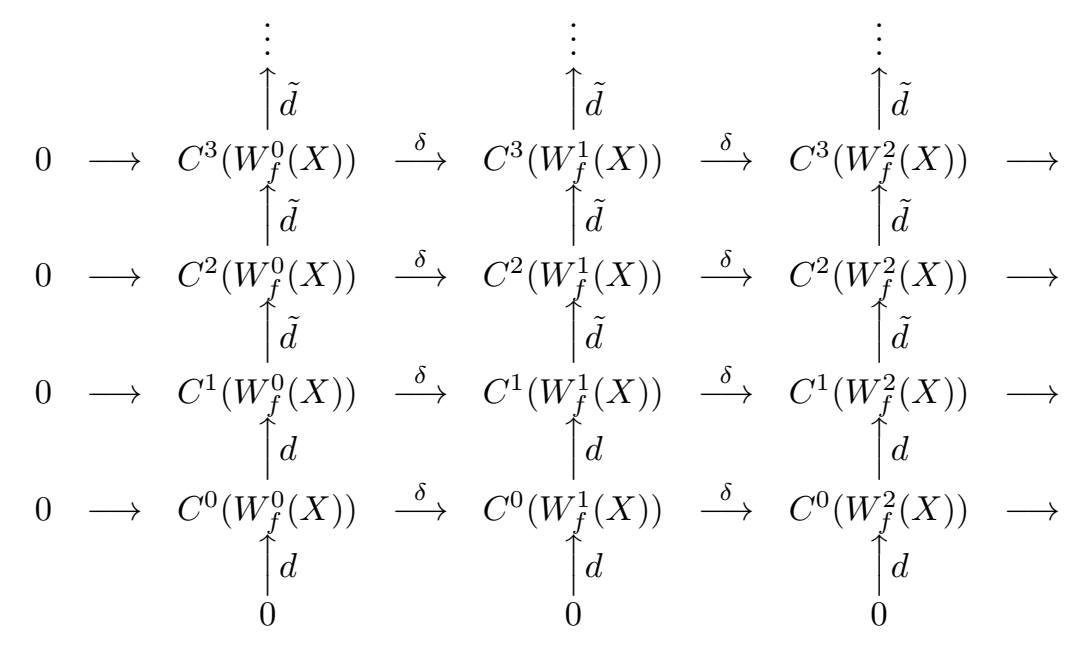

Lemma 5.4. The families of maps $\tilde{d}$ and $\delta$ make $D^{\bullet \bullet \bullet}$ into a double complex.

Proof. We need to check that $\tilde{d}^{2}=\delta^{2}=\tilde{d} \delta+\delta \tilde{d}=0$. We know that $\tilde{d}^{2}=d^{2}=0$ since $C^{\bullet}\left(W_{f}^{p}(X)\right)$ is a cochain complex for all $p$, and we proved that $\delta^{2}=0$ in Proposition 5.3

Now, suppose that $\phi \in C^{q}\left(W_{f}^{p}(X)\right)$ and let $s=\left(s_{0}, \ldots, s_{p+1}\right)$ be an admissible singular $(q+1)$-simplex of $W_{f}^{p+1}(X)$. Then,

$$
\begin{aligned}
\tilde{d}(\delta \phi)(s) & =\tilde{d}\left(\sum_{i=0}^{p+1}(-1)^{i} \phi\left(\left(s_{0}, \ldots, \widehat{s_{i}}, \ldots, s_{p+1}\right)\right)\right) \\
& =(-1)^{p} \sum_{j=0}^{q+1} \sum_{i=0}^{p+1}(-1)^{i+j} \phi\left(s_{0} \circ f_{q, j}, \ldots, \widehat{s_{i} \circ f_{q}, j}, \ldots, s_{p+1} \circ f_{q, j}\right) .
\end{aligned}
$$

We also have

$$
\begin{aligned}
\delta(\tilde{d} \phi)(s) & =\delta\left((-1)^{p+1} \sum_{j=0}^{q+1}(-1)^{j} \phi\left(s_{0} \circ f_{q, j}, \ldots, s_{p+1} \circ f_{q, j}\right)\right) \\
& =(-1)^{p+1} \sum_{j=0}^{q+1} \sum_{i=0}^{p+1}(-1)^{i+j} \phi\left(s_{0} \circ f_{q, j}, \ldots, \widehat{s_{i} \circ f_{q, j}}, \ldots, s_{p+1} \circ f_{q, j}\right) .
\end{aligned}
$$

Thus, it follows that $\tilde{d} \delta+\delta \tilde{d}=0$, so $D^{\bullet \bullet \bullet}$ is indeed a double complex.

If $f: X \rightarrow Y$ is locally split, and if $\mathcal{V}$ is the corresponding open covering of $X$ defined in Section 5.1 the double complex $D^{\bullet \bullet \bullet}$ induces by restriction a double complex $D_{\mathcal{V}}^{\bullet \bullet}$, where $D_{\mathcal{V}}^{p, q}=C_{\mathcal{V}}^{q}\left(W_{f}^{p}(X)\right)$ when $p \geq 0$ and $q \geq 0$ and $D_{\mathcal{V}}^{\bullet, \bullet}=0$ otherwise. 
The initial terms of the two spectral sequences associated with $D_{\mathcal{V}}^{\bullet \bullet}$ (cf. Section (3.3) are as follows. The first terms of the spectral sequence ${ }^{\prime} E_{*}^{i, j}$ are ${ }^{\prime} E_{1}=$ $H_{\delta}\left(D_{\mathcal{V}}^{\bullet \bullet \bullet}(X)\right),{ }^{\prime} E_{2}=H_{\tilde{d}} H_{\delta}\left(D_{\mathcal{V}}^{\bullet \bullet}(X)\right)$. By the exactness of the sequence in Proposition [5.3] we have that the spectral sequence ${ }^{\prime} E_{*}^{i, j}$ degenerates at the ${ }^{\prime} E_{2}$ term as shown below.

$$
{ }^{\prime} E_{1}=\mid \begin{array}{cccccc}
\vdots & \vdots & \vdots & \vdots & \vdots & \\
\uparrow d & \uparrow 0 & \uparrow 0 & \uparrow 0 & \uparrow 0 & \\
C_{\mathcal{V}}^{3}(Y) & 0 & 0 & 0 & 0 & \ldots \\
\uparrow d & \uparrow 0 & \uparrow 0 & \uparrow 0 & \uparrow 0 & \\
C_{\mathcal{V}}^{2}(Y) & 0 & 0 & 0 & 0 & \ldots \\
\uparrow d & \uparrow 0 & \uparrow 0 & \uparrow 0 & \uparrow 0 & \\
C_{\mathcal{V}}^{1}(Y) & 0 & 0 & 0 & 0 & \ldots \\
\uparrow d & \uparrow 0 & \uparrow 0 & \uparrow 0 & \uparrow 0 & \\
C_{\mathcal{V}}^{0}(Y) & 0 & 0 & 0 & 0 & \ldots
\end{array}
$$

and, by Proposition 5.2

$$
{ }^{\prime} E_{2}=\begin{array}{ccccccc}
\vdots & \vdots & \vdots & \vdots & \vdots & \vdots & \\
H^{3}(Y) & 0 & 0 & 0 & 0 & 0 & \cdots \\
H^{2}(Y) & 0 & 0 & 0 & 0 & 0 & \cdots \\
H^{1}(Y) & 0 & 0 & 0 & 0 & 0 & \cdots \\
H^{0}(Y) & 0 & 0 & 0 & 0 & 0 & \cdots
\end{array}
$$

The degeneration of this sequence at ' $E_{2}$ shows that

$$
H^{*}\left(\operatorname{Tot}^{\bullet}\left(D_{\mathcal{V}}^{\bullet \bullet}(X)\right)\right) \cong H^{*}(Y) .
$$

The initial term " $E_{1}$ of the second spectral sequence is given by,

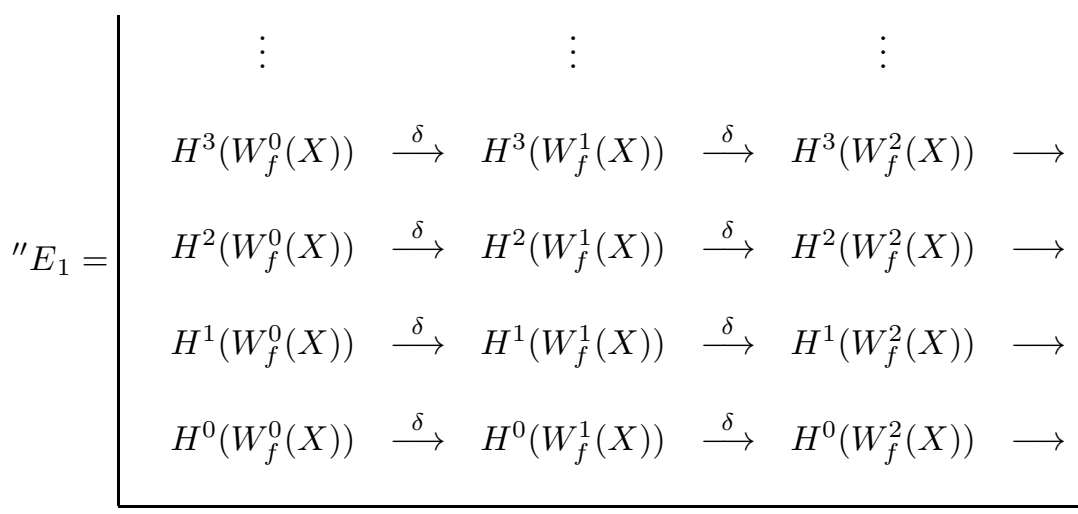


Since this spectral sequence also converges to $H^{*}\left(\operatorname{Tot}^{\bullet}\left(D_{\mathcal{V}}^{\bullet \bullet \bullet}\right)(X)\right)$, we have the following proposition.

\section{Proposition 5.5.}

$$
H^{*}\left(\operatorname{Tot}^{\bullet}\left(D_{\mathcal{V}}^{\bullet \bullet}\right)(X)\right) \cong H^{*}(Y)
$$

Proposition 5.5 now implies,

Theorem 5.6. For any continuous semi-algebraic surjection $f: X \rightarrow Y$, where $X$ and $Y$ are open semi-algebraic subsets of $\mathbb{R}^{n}$ and $\mathbb{R}^{m}$ respectively (or, more generally, for any locally split continuous surjection $f$ ), the spectral sequence associated to the double complex $D^{\bullet \bullet \bullet}(X)$ with $E_{1}=H_{d}\left(D^{\bullet \bullet \bullet}(X)\right)$ converges to $H^{*}\left(C^{\bullet}(Y)\right) \cong H^{*}(Y)$. In particular,

(A) $E_{1}^{i, j}=H^{j}\left(W_{f}^{i}(X)\right)$, and

(B) $E_{\infty} \cong H^{*}\left(\operatorname{Tot}^{\bullet}\left(D^{\bullet \bullet} \bullet(X)\right)\right) \cong H^{*}(Y)$.

Proof. By Proposition 5.2 we have that the component-wise homomorphisms, $\iota^{\bullet}$, induces a homomorphism of double complexes,

$$
\iota^{\bullet \bullet \bullet}: D^{\bullet, \bullet} \rightarrow D_{\mathcal{V}}^{\bullet \bullet}
$$

which in turn induces an isomorphism between the $E_{1}$ terms of the corresponding spectral sequences. Hence, by Theorem 3.1 we have that, $H^{*}\left(\operatorname{Tot}^{\bullet}\left(D_{\mathcal{V}}^{\bullet, \bullet}\right)\right) \cong$ $H^{*}\left(\operatorname{Tot}^{\bullet}\left(D^{\bullet, \bullet}\right)\right)$. The Theorem now follows from Proposition 5.5

5.4. Truncation of the double complex. If we denote by $D_{q}^{\bullet \bullet \bullet}(X)$ the truncated complex defined by,

$$
\begin{aligned}
& D_{q}^{i, j}(X)=D^{i, j}(X), \quad \text { if } \quad 0 \leq i+j \leq q+1, \\
& =0, \quad \text { otherwise, }
\end{aligned}
$$

then it is clear that,

$$
H^{i}(Y) \cong H^{i}\left(\operatorname{Tot}^{\bullet}\left(D_{q}^{\bullet \bullet \bullet}(X)\right)\right), \text { for } \quad 0 \leq i \leq q .
$$

Now suppose that $X \subset \mathbb{R}^{k+m}$ is a compact semi-algebraic set defined by the inequalities, $P_{1} \geq 0, \ldots, P_{\ell} \geq 0$. Let $\pi$ denote the projection map, $\pi: \mathbb{R}^{k+m} \rightarrow \mathbb{R}^{m}$. Let $\epsilon>0$ and let $\tilde{X} \subset \mathbb{R}^{k+m}$ be the set defined by $P_{1}+\epsilon>0, \ldots, P_{\ell}+\epsilon>0$.

Proposition 5.7. $\quad$ (A) For $\epsilon>0$ sufficiently small, we have

$$
\begin{gathered}
H^{*}\left(W_{\pi}^{p}(\tilde{X})\right) \cong H^{*}\left(W_{\pi}^{p}(X)\right), \text { for all } p \geq 0, \\
\text { and } H^{*}(\pi(\tilde{X})) \cong H^{*}(\pi(X)) .
\end{gathered}
$$

(B) The map, $\left.\pi\right|_{\tilde{X}}$ is a locally split semi-algebraic surjection onto its image.

Proof. When $\epsilon>0$ is small, the sets $X$ and $\tilde{X}$ are homotopy equivalent and so are the sets $\pi(X)$ and $\pi(\tilde{X})$ and the fibered products $W_{\pi}^{p}(\tilde{X})$ and $W_{\pi}^{p}(X)$ for all $p \geq 0$ (see [4). The first part of the proposition follows from the homotopy invariance property of singular cohomology groups. The second part of the proposition is clear once we note that $\tilde{X}$ is an open subset of $\mathbb{R}^{k+m}$ : projections of open sets always admit local continuous sections.

We can combine Theorem 5.6 and Proposition 5.7 to construct, from the projection of a compact basic semi-algebraic set, a double complex giving rise to a cohomological descent spectral sequence. 
Corollary 5.8. Let $X \subset \mathbb{R}^{k+m}$ be a compact semi-algebraic set defined by $P_{1} \geq$ $0, \ldots, P_{\ell} \geq 0$ and $\pi: R^{k+m} \rightarrow \mathbb{R}^{m}$ the projection onto the last $m$ co-ordinates. The spectral sequence associated to the double complex $D^{\bullet \bullet \bullet}(X)$ with $E_{1}=H_{d}\left(D^{\bullet \bullet \bullet}(X)\right)$ converges to $H^{*}\left(C^{\bullet}(\pi(X))\right) \cong H^{*}(\pi(X))$. In particular,

(A) $E_{1}^{i, j}=H^{j}\left(W_{f}^{i}(X)\right)$, and

(B) $E_{\infty} \cong H^{*}\left(\operatorname{Tot}^{\bullet}\left(D^{\bullet \bullet \bullet}(X)\right)\right) \cong H^{*}(\pi(X))$.

Remark 5.9. Note that it is not obvious how to prove directly an exact sequence at the level of singular (or even simplicial) cochains for the projection of a compact set, as we do in Proposition 5.3 in the locally-split setting. One difficulty is the fact that semi-algebraic maps are not, in general, triangulable.

Now let $X$ be a compact semi-algebraic set defined by a constant number of quadratic inequalities and $f$ a projection map. We cannot hope to compute even the truncated complex $D_{q}^{\bullet \bullet}(X)$ since these are defined in terms of singular chain complexes which are infinite-dimensional. We overcome this problem by computing another double complex $\mathcal{D}_{q}^{\bullet \bullet \bullet}(X)$, such that there exists a homomorphism of double complexes, $\psi: \mathcal{D}_{q}^{\bullet \bullet \bullet}(X) \longrightarrow D_{q}^{\bullet, \bullet}(X)$, which induces an isomorphism between the ${ }^{\prime} E_{1}$ terms of the spectral sequences associated to the double complexes $D_{q}^{\bullet \bullet \bullet}(X)$ and $\mathcal{D}_{q}^{\bullet \bullet \bullet}(X)$. This implies, by virtue of Theorem 3.1 that the cohomology groups of the associated total complexes are isomorphic, that is,

$$
H^{*}\left(\operatorname{Tot}^{\bullet}\left(D_{q}^{\bullet \bullet \bullet}(X)\right)\right) \cong H^{*}\left(\operatorname{Tot}^{\bullet}\left(\mathcal{D}_{q}^{\bullet, \bullet}(X)\right)\right) .
$$

The construction of the double complex $\mathcal{D}_{q}^{\bullet \bullet \bullet}(X)$ is described in Section $\mathbf{Z}$

\section{Algorithmic Preliminaries}

We now recall an algorithm described in [7, where the following theorem is proved.

Theorem 6.1. There exists an algorithm, which takes as input a family of polynomials $\left\{P_{1}, \ldots, P_{s}\right\} \subset \mathbb{R}\left[X_{1} \ldots, X_{k}\right]$, with $\operatorname{deg}\left(P_{i}\right) \leq 2$, and a number $\ell \leq k$, and outputs a complex $\mathcal{D}_{\ell}^{\bullet, \bullet}$. The complex $\operatorname{Tot}^{\bullet}\left(\mathcal{D}_{\ell}^{\bullet, \bullet}\right)$ is quasi-isomorphic to $\mathrm{C}_{\bullet}^{\ell}(S)$, the truncated singular chain complex of $S$, where

$$
S=\bigcap_{P \in \mathcal{P}}\left\{x \in \mathbb{R}^{k} \mid P(x) \leq 0\right\} .
$$

Moreover, given a subset $\mathcal{P}^{\prime} \subset \mathcal{P}$, with

$$
S^{\prime}=\bigcap_{P \in \mathcal{P}^{\prime}}\left\{x \in \mathbb{R}^{k} \mid P(x) \leq 0\right\} .
$$

the algorithm outputs both complexes $\mathcal{D}_{\ell}^{\bullet, \bullet}$ and $\mathcal{D}_{\ell}^{\prime \bullet, \bullet}$ (corresponding to the sets $S$ and $S^{\prime}$ respectively) along with the matrices defining a homomorphism $\Phi_{\mathcal{P}, \mathcal{P}^{\prime}}$, such that $\Phi_{\mathcal{P}, \mathcal{P}^{\prime}}^{*}: H^{*}\left(\operatorname{Tot}^{\bullet}\left(\mathcal{D}_{\ell}^{\bullet, \bullet}\right)\right) \cong H^{*}(S) \rightarrow H^{*}\left(S^{\prime}\right) \cong H^{*}\left(\operatorname{Tot}^{\bullet}\left(\mathcal{D}_{\ell}^{\prime \bullet \bullet \bullet}\right)\right)$ is the homomorphism induced by the inclusion $i: S \hookrightarrow S^{\prime}$. The complexity of the algorithm is $\sum_{i=0}^{\ell+2}\left(\begin{array}{c}s \\ i\end{array}\right) k^{2^{O(\min (\ell, s))}}$.

For completeness, we formally state the input and output of the algorithm mentioned in Theorem 6.1

We first introduce some notations which will be used to describe the input and output of the algorithm. Let $\mathcal{Q}=\left\{Q_{1}, \ldots, Q_{s}\right\} \subset \mathbb{R}\left[X_{1}, \ldots, X_{k}\right]$ be a family of polynomials with $\operatorname{deg}\left(Q_{i}\right) \leq 2,1 \leq i \leq s$. For each subset $J \subset\{1, \ldots, s\}$, we let 
$S_{J}$ denote the semi-algebraic set defined by $\left\{Q_{j} \geq 0 \mid j \in J\right\}$. Notice that for each pair $I \subset J \subset\{1, \ldots, s\}$, we have an inclusion $S_{J} \subset S_{I}$.

Algorithm 1 (Build Complex).

Input: A family of polynomials $\mathcal{Q}=\left\{Q_{1}, \ldots, Q_{s}\right\} \subset \mathbb{R}\left[X_{1}, \ldots, X_{k}\right]$ with $\operatorname{deg}\left(Q_{i}\right) \leq$ 2 , for $1 \leq i \leq s$.

\section{Output:}

(A) For each subset $J \subset\{1, \ldots, s\}$, a description of a complex $F_{J}^{\bullet}$, consisting of a basis for each term of the complex and matrices (in this basis) for the differentials, and

(B) for each pair $I \subset J \subset\{1, \ldots, s\}$, a homomorphism, $\phi_{I, J}: F_{I}^{\bullet} \longrightarrow F_{J}^{\bullet}$.

The complexes, $F_{J}^{\bullet}$ and the homomorphisms $\phi_{I, J}$ satisfy the following.

(A) For each $J \subset\{1, \ldots, s\}$,

$$
H^{*}\left(F_{J}^{\bullet} \cong H^{*}\left(S_{J}\right)\right.
$$

(B) For each pair $I \subset J \subset\{1, \ldots, s\}$, the following diagram commutes.

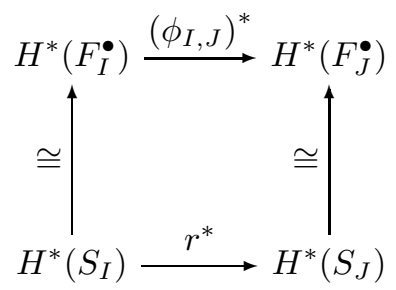

Here, $\left(\phi_{I, J}\right)^{*}$ is the homomorphism induced by $\phi_{I, J}$, the vertical homomorphisms are the isomorphisms from (6.1), and $r^{*}$ is the homomorphism induced by restriction.

Complexity: The complexity of the algorithm is $k^{2^{O(s)}}$.

For the purposes of this paper, we need to slightly modify Algorithm 1 in order to be able to handle permutations of the co-ordinates. More precisely, suppose that $\sigma \in \mathfrak{S}_{k}$ is a given permutation of the co-ordinates, and for any $I \subset\{1, \ldots, s\}$, let $S_{I, \sigma}=\left\{\left(x_{\sigma(1)}, \ldots, x_{\sigma(k)}\right) \mid\left(x_{1}, \ldots, x_{k}\right) \in S_{I}\right\}$. Let $F_{I, \sigma}^{\bullet}$ denote the complex computed by the algorithm corresponding to the set $S_{I, \sigma}$. It is easy to modify Algorithm 1 slightly without changing the complexity estimate, such that for any fixed $\sigma$, the algorithm outputs, complexes $F_{I}^{\bullet}, F_{I, \sigma}^{\bullet}$ as well as the matrices corresponding to the induced isomorphisms, $\phi_{\sigma}^{\bullet}: F_{I}^{\bullet} \rightarrow F_{I, \sigma}^{\bullet}$. We assume this implicitly in the description of Algorithm [2] in the next section.

\section{Algorithm For Projections}

Let $S \subset \mathbb{R}^{k+m}$ be a basic semi-algebraic set defined by

$$
P_{1} \geq 0, \ldots, P_{\ell} \geq 0, P_{i} \in \mathbb{R}\left[X_{1}, \ldots, X_{k}, Y_{1}, \ldots, Y_{m}\right],
$$

with $\operatorname{deg}\left(P_{i}\right) \leq 2,1 \leq i \leq \ell$. Let $\pi: \mathbb{R}^{k+m} \rightarrow \mathbb{R}^{m}$ be the projection onto the last $m$ coordinates.

The algorithm will compute a double complex, $\mathcal{D}_{q}^{\bullet \bullet \bullet}(S)$, such that $\operatorname{Tot}^{\bullet}\left(\mathcal{D}_{q}^{\bullet \bullet \bullet}(S)\right)$ is quasi-isomorphic to the $\operatorname{complex}^{\operatorname{Tot}^{\bullet}}\left(D_{q}^{\bullet, \bullet}(S)\right)$. The double complex, $\mathcal{D}_{q}^{\bullet, \bullet}(S)$ is defined as follows. 
We introduce $k(q+2)$ variables, which we denote by $X_{i, j}, 1 \leq i \leq k, 0 \leq j \leq q+1$. For each $j, 0 \leq j \leq q+1$, we denote by, $P_{i, j}$ the polynomial

$$
P_{i}\left(X_{1, j}, \ldots, X_{k, j}, Y_{1}, \ldots, Y_{m}\right)
$$

(substituting $X_{1, j}, \ldots, X_{k, j}$ in place of $X_{1}, \ldots, X_{k}$ in the polynomial $P_{i}$ ). We consider each $P_{i, j}$ to be an element of $\mathbb{R}\left[X_{1,0}, \ldots, X_{k, q+1}, Y_{1}, \ldots, Y_{m}\right]$. For each $p, 0 \leq p \leq q+1$, we denote by $S_{p} \subset \mathbb{R}^{k(q+2)+m}$ the semi-algebraic set defined by,

$$
P_{1,0} \geq 0, \ldots, P_{\ell, 0} \geq 0, \ldots, P_{1, p} \geq 0, \ldots, P_{\ell, p} \geq 0 .
$$

Note that, for each $p, 0<p \leq q+1$, and each $j, 0 \leq j \leq p$ we have a natural map, $\pi_{p, j}: S_{p} \rightarrow S_{p-1}$ given by,

$$
\pi_{p, j}\left(\bar{x}_{0}, \ldots, \bar{x}_{p}, \ldots, \bar{x}_{q+1}, \bar{y}\right)=\left(\bar{x}_{0}, \ldots, \bar{x}_{p}, \ldots, \bar{x}_{j}, \ldots, \bar{x}_{q+1}, \bar{y}\right) .
$$

Note that in the definition above, each $\bar{x}_{i} \in \mathbb{R}^{k}$ and $\pi_{p, j}$ exchanges the coordinates $\bar{x}_{j}$ and $\bar{x}_{p}$.

We are now in a position to define $\mathcal{D}_{q}^{\bullet \bullet \bullet}$. We follow the notations introduced in Section [6] Let $\mathcal{Q}=\left\{Q_{1}, \ldots, Q_{\ell(q+2)}\right\}=\left\{P_{1,0}, \ldots, P_{\ell, q+1}\right\}$. For $0 \leq j \leq q+1$, we let $L_{j}=\{1, \ldots,(j+1) \ell\} \subset\{1, \ldots,(q+2) \ell\}$.

$$
\begin{array}{rlc}
\mathcal{D}_{q}^{i, j}(X) & =F_{L_{i}}^{j}, & 0 \leq i+j \leq q+1, \\
& =0, & \text { otherwise }
\end{array}
$$

The vertical homomorphisms, $d$, in the complex $\mathcal{D}_{q}^{\bullet \bullet \bullet}$ are those induced from the complexes $F_{L_{i}}^{\bullet}$ or zero. The horizontal homomorphisms, $\delta^{j}: F_{L_{i}}^{j} \longrightarrow F_{L_{i+1}}^{j}$ are defined as follows.

For each $h, 0 \leq h \leq i+1$, Algorithm 1 produces a homomorphism, $\phi_{i+1, h}$ : $F_{L_{i}}^{j} \longrightarrow F_{L_{i+1}}^{j}$, corresponding to the map $\pi_{i+1, h}$ (see remark after Algorithm 1). The homomorphism $\delta$ is then defined by, $\delta=\sum_{h=0}^{i+1}(-1)^{h} \phi_{i+1, h}$. We have the following proposition.

Proposition 7.1. The complex $\operatorname{Tot}^{\bullet}\left(\mathcal{D}_{q}^{\bullet \bullet}(S)\right)$ is quasi-isomorphic to the complex $\operatorname{Tot}^{\bullet}\left(D_{q}^{\bullet \bullet}(S)\right)$.

Proof. It follows immediately from Theorem 6.1 that the columns of the complexes $\mathcal{D}_{q}^{\bullet, \bullet}(S)$ and $D_{q}^{\bullet \bullet \bullet}(S)$ are quasi-isomorphic. Moreover, it is easy to see that the quasiisomorphisms induce an isomorphism between the " $E_{1}$ term of their associated spectral sequences. Now by Theorem 3.1 this implies that $\operatorname{Tot}^{\bullet}\left(\mathcal{D}_{q}^{\bullet \bullet \bullet}(S)\right)$ is quasiisomorphic to the complex $\operatorname{Tot}^{\bullet}\left(D_{q}^{\bullet, \bullet}(S)\right)$.

Algorithm 2 (Computing the first $q$ Betti Numbers).

Input: A $S \subset \mathbb{R}^{k+m}$ be a basic semi-algebraic set defined by

$$
P_{1} \geq 0, \ldots, P_{\ell} \geq 0
$$

with $P_{i} \in \mathbb{R}\left[X_{1}, \ldots, X_{k}, Y_{1}, \ldots, Y_{m}\right], \operatorname{deg}\left(P_{i}\right) \leq 2,1 \leq i \leq \ell$.

Output: $b_{0}(\pi(S)), \ldots, b_{q}(\pi(S))$, where $\pi: \mathbb{R}^{k+m} \rightarrow \mathbb{R}^{m}$ be the projection onto the last $m$ coordinates.

\section{Procedure:}

Step 1: Using Algorithm 1 compute the truncated complex $\mathcal{D}_{q}^{\bullet \bullet \bullet}(S)$.

Step 2: Compute using linear algebra, the dimensions of $H^{i}\left(\operatorname{Tot}^{\bullet}\left(\mathcal{D}_{q}^{\bullet, \bullet}\right)\right), \quad 0 \leq i \leq q$. Step 3: For each $i, \quad 0 \leq i \leq q$, output, $b_{i}(\pi(S))=\operatorname{dim}\left(H^{i}\left(\operatorname{Tot}^{\bullet}\left(\mathcal{D}_{q}^{\bullet \bullet \bullet}\right)\right)\right)$. 
Complexity Analysis: The calls to Algorithm 1 has input consisting of $(q+1) \ell$ polynomials in $q k+m$ variables. Using the complexity bound of Algorithm 1 we see that the complexity of Algorithm 2 is bounded by $(k+m)^{2^{O(q \ell)}}$

Proof of Correctness: The correctness of the algorithm is a consequence of Proposition 7.1 and Theorem 3.1 .

\section{Conclusion and Open Problems}

For any fixed $q$ and $\ell$, we have proved a polynomial bound on the sum of the first $q$ Betti numbers of the projection of a bounded, basic closed semi-algebraic set defined by $\ell$ quadratic inequalities. We have also described a polynomial time algorithm to compute the first $q$ Betti numbers of the image of such a projection.

Since it is not known whether quantifier elimination can be performed efficiently for sets defined by a fixed number of quadratic inequalities, many questions are left open.

Our bounds become progressively worse as $q$ increases, becoming exponential in the dimension as $q$ approaches $k$. However, we do not have any examples (of projections of semi-algebraic sets defined by quadratic inequalities) where the higher Betti numbers behave exponentially in the dimension. This leaves open the problem of either constructing such examples, or removing the dependence on $q$ from our bounds.

Another interesting open problem is to improve the complexity of Algorithm 2 from $(k+m)^{2^{O(q \ell)}}$ to $(k+m)^{O(q \ell)}$. Note that this would imply an algorithm with complexity $k^{O(q \ell)}$ for computing the first $q$ Betti numbers of a semi-algebraic set defined by $\ell$ quadratic inequalities in $\mathbb{R}^{k}$. The best known algorithm for computing all the Betti numbers of such sets has complexity $k^{2^{O(\ell)}}$ [7. The only topological invariants of such sets that we currently know how to compute in time $k^{O(\ell)}$ are testing for emptiness [2, 21] and the Euler-Poincaré characteristic [9].

\section{REFERENCES}

[1] A. A. Agrachev, Topology of quadratic maps and Hessians of smooth maps, Algebra, Topology, Geometry, Vol 26 (Russian),85-124, 162, Itogi Nauki i Tekhniki, Akad. Nauk SSSR, Vsesoyuz. Inst. Nauchn.i Tekhn. Inform., Moscow, 1988. Translated in J. Soviet Mathematics. 49 (1990), no. 3, 990-1013.

[2] A. I. Barvinok, Feasibility Testing for Systems of Real Quadratic Equations, Discrete and Computational Geometry, 10:1-13 (1993).

[3] A. I. BARVINOK On the Betti numbers of semialgebraic sets defined by few quadratic inequalities Math. Zeit., 225:231-244, 1997.

[4] S. BAsu, On Bounding the Betti Numbers and Computing the Euler Characteristics of Semialgebraic Sets, Discrete and Computational Geometry, 22 1-18 (1999).

[5] S. Basu, R. Pollack, M.-F. Roy, On the Combinatorial and Algebraic Complexity of Quantifier Elimination, Journal of the ACM, 43 1002-1045, (1996).

[6] S. BAsu, On different bounds on different Betti numbers, Discrete and Computational Geometry, 30:1, 65-85, 2003.

[7] S. BAsu, Polynomial time algorithm for computing the top Betti numbers of Semi-algebraic sets defined by quadratic inequalities, Proceedings of Symposium on the Theory of Computing, 2005. Available at www.math.gatech.edu/ saugata/quadratic.ps.

[8] S. BASU, Single exponential time algorithm for computing the first few Betti numbers of semi-algebraic sets, preprint. Available at www.math.gatech.edu/ saugata/bettifew.ps

[9] S. BASU, Efficient algorithm for computing the Euler-Poincaré characteristic of semialgebraic sets defined by few quadratic inequalities, to appear in Computational Complexity. Available at www.math.gatech.edu/ saugata/eulerquad.pdf 
[10] S. Basu, R. Pollack, M.-F. Roy, Computing the first Betti number and the connected components of semi-algebraic sets, Proceedings of Symposium on the Theory of Computing, 2005 (to appear). Available at www.math.gatech.edu/ saugata/bettione.ps.

[11] S. Basu, R. Pollack, M.-F. Roy, Algorithms in Real Algebraic Geometry, Springer-Verlag, 2003.

[12] J. Bochnak, M. Coste, M.-F. Roy, Géométrie algébrique réelle, Springer-Verlag (1987). Real algebraic Geometry, Springer-Verlag (1998).

[13] R. Bотt, L. W. Tu, Differential Forms in Algebraic Topology, Springer-Verlag (1982).

[14] P. Burgisser, F. CuCKer, Counting Complexity Classes for Numeric Computations II: Algebraic and Semi-algebraic Sets, preprint.

[15] G. E. Bredon, Sheaf Theory, Springer-Verlag (1996).

[16] G. Collins, Quantifier elimination for real closed fields by cylindric algebraic decomposition, In Second GI Conference on Automata Theory and Formal Languages. Lecture Notes in Computer Science, vol. 33, pp. 134-183, Springer-Verlag, Berlin (1975).

[17] P. Deligne, Théorie de Hodge III, Publ. Math. IHES 44:5-77, 1974.

[18] D. Dugger and D. Isaksen, Topological hypercovers and $A^{1}$-realizations, Math. Z 246 (2004), $667-689$.

[19] A. Gabrielov, N. Vorobjov, T. Zell, Betti Numbers of Semi-algebraic and Sub-Pfaffian Sets, J. London Math. Soc. (2) 69 (2004) 27-43.

[20] A. GABRielov, Counter-examples to quantifier elimination for fewnomial and exponential expressions, preprint. Available at http://www.math.purdue.edu/ agabriel/preprint.html

[21] D. Grigor'ev, D.V. Pasechnik, Polynomial time computing over quadratic maps I. Sampling in real algebraic sets, Computational Complexity 14:20-52, (2005).

[22] R. M. HARdt, Semi-algebraic Local Triviality in Semi-algebraic Mappings, Am. J. Math. 102, 291-302 (1980).

[23] A. Hatcher, Algebraic Topology, Cambridge University Press (2002).

[24] K. Houston, An introduction to the image computing spectral sequence, In Singularity theory (Liverpool, 1996), volume 263 of London Math. Soc. Lecture Note Ser., pages 305-324. Cambridge Univ. Press, Cambridge, 1999.

[25] A. G. Khovansky Fewnomials, American Mathematical Society, 1991.

[26] J. McCleary A User's Guide to Spectral Sequences, Second Edition Cambridge Studies in Advanced Mathematics, 2001.

[27] J. Milnor, On the Betti numbers of real varieties, Proc. AMS 15, 275-280 (1964).

[28] M. Murray, Bundle gerbes, J. London Math. Soc. 54, 403-416 (1996).

[29] O. A. OleĬNIK, Estimates of the Betti numbers of real algebraic hypersurfaces, Mat. Sb. (N.S.), 28 (70): 635-640 (Russian) (1951).

[30] O. A. OleıñK, I. B. Petrovskit, On the topology of real algebraic surfaces, Izv. Akad. Nauk SSSR 13, 389-402 (1949).

[31] J. RENEGAR. On the computational complexity and geometry of the first order theory of the reals, Journal of Symbolic Computation, 13: 255-352 (1992).

[32] B. Saint-Donat, Techniques de descente cohomologique, In Théorie des topos et cohomologie étale des schémas. Tome 2, Springer-Verlag, Berlin, 1972 (SGA 4), Lecture Notes in Mathematics, Vol. 270, p. 83-162.

[33] R. Тном, Sur l'homologie des variétés algébriques réelles, Differential and Combinatorial Topology, 255-265. Princeton University Press, Princeton (1965).

[34] V. VAssiliev, Complements of discriminants of smooth maps: topology and applications, volume 98 of Translations of Mathematical Monographs. American Mathematical Society, Providence, RI, 1992.

[35] T. ZeLL, Topology of definable Hausdorff limits, Discrete Comput. Geom. 33, 423-443 (2005).

School of Mathematics, Georgia Institute of Technology, Atlanta, Ga 30332, U.S.A. E-mail address: saugata@math.gatech.edu

School of Mathematics, Georgia Institute of Technology, Atlanta, Ga 30332, U.S.A.

E-mail address: zell@math.gatech.edu 